\title{
Imaging appearance and role of CEUS of high flow priapism. A case report.
}

\author{
Florin Elec ${ }^{1}$, Tudor Moisoiu', Burghelea Dan', Razvan Zaro², Radu Badea ${ }^{2}$ \\ ${ }^{1}$ Clinical Institute of Urology and Kidney Transplantation, ${ }^{2}$ Ultrasound Imaging Department, "Octavian Fodor" \\ Regional Institute of Gastroenterology and Hepatology, "Iuliu Hatieganu" University of Medicine and Pharmacy, \\ Cluj-Napoca, Romania
}

\begin{abstract}
High flow priapism caused by perineal trauma is a relatively rare disorder. Early diagnosis represents a mandatory condition for the therapeutic resolution. Ultrasound examination is affordable and a within reach method for diagnosis also in an emergency context. We present the case of a 56-year-old male patient with traumatic priapism which was subsequently investigated by contrast-enhanced ultrasound and shear wave elastography. This may be one of the first cases presented in the literature.
\end{abstract}

Keywords: priapism; imaging; contrast enhanced ultrasonography (CEUS)

\section{Introduction}

Priapism represents a disorder of penile erection that persists or is unrelated to sexual stimulation [1]. Arterial priapism (high flow) is a pathological condition caused by perineal or penile blunt trauma and formation of an arterial-lacunar fistula, triggering an unregulated cavernous arterial inflow [2]. The manifestation of this condition is represented by not fully rigid erection without pain [3]. We present the case of a 56-year-old man with an arterial priapism neglected for 3 weeks with extensive imaging investigation, including contrast-enhanced ultrasonography (CEUS).

Received 10.01.2019 Accepted 30.01.2019

Med Ultrason

2019, Vol. 21, No 3, 353-355

Corresponding author: Răzvan Zaro

Ultrasound Imaging Department,

"Octavian Fodor" Regional Institute of

Gastroenterology and Hepatology

19-21 Croitorilor Street,

400162, Cluj Napoca, România

E-mail: Razvan.Zaro@umfcluj.ro

\section{Case report}

A 56-year-old man presented with a 3-week history of painless penile erection due to a perineal trauma during manipulation of hubs. At presentation, the patient had an episode of hypertension and supraventricular tachycardia prior to any procedure that remitted after specific therapy. The patient had no history of other pathology or drug abuse. Early imaging workup was ultrasonography in grayscale followed by Doppler ultrasound, showing turbulent flow at the site of a hypoechogenic area; penile blood analysis was suggesting arterial blood. Contrast enhanced magnetic resonance imaging (CE-MRI) confirmed the diagnosis of arterial priapism. Figure 1 (a) and (b) reproduces the dilacerations of lacunar tissue at the base of left corpus cavernosum and shows fibrous tissue starting to form.

Treatment options were restricted to a conservative approach because selective arterial embolization was not available in our radiologic department. Surgical ligation of the pudendal artery at 3 weeks after the trauma, having a very low rate of success, was refused by the patient. Local ice was applied with partial relief of the erection. 
Also, antibiotic therapy was administered to prevent the infection of lacerated tissue. The patient was informed about the high incidence of impotence due to fibrotic tissue.

At the 2 weeks follow-up the patient had a partial remission of erection with no other complaints. At this time an ultrasound (US) evaluation was performed. We used a Logiq E9-GE machine (General Electric Healthcare, Boston, USA) and a linear transducer (L9) with a frequency of $7.5 \mathrm{MHz}$. The patient was positioned supine and predominantly transverse sections at the base of the cavernous bodies were obtained in order to detect the area of interest considered to be relevant for the pathology. US grayscale examination strengthens the clinical suspicion of thrombosis of the cavernous bodies. Also, high-resolution US revealed a discreetly hypoechogenic area at the level of the left cavernous body with turbulent flow and arterial signal evidenced by the Doppler Color Flow Map (CFM) (fig 2) For CEUS examination a quantity of $2.4 \mathrm{ml}$ of contrast agent (Sonovue, Bracco) was injected and the mechanical index was set to 0.14. By CEUS, during arterial phase (0-30 seconds) a peripheral vascular signal was highlighted as a semi-circumferential capture through branches from the dorsal arteries and a vascular tract inside the left cavernous body - apparently "blind" with a turbulent pattern at this level was found. The CEUS appearance from the rending area of the cavernous body was maintained up to 120 seconds suggesting a possible vascular lake (fig $3 a, b$ ).

Comparative evaluation by shear wave elastography technique (SWE) revealed a non-homogeneous aspect of the dilaceration area, suggesting the intralesional fibrous reshaping process in the left cavernous body. This aspect is highlighted by the non-homogeneous pattern and the rigidity at this level measured at $27.30 \mathrm{kPa}$ versus the contralateral cavernous body with a homogeneous aspect and a rigidity of $13.15 \mathrm{kPa}$ (fig $3 \mathrm{c}, \mathrm{d}$ ).

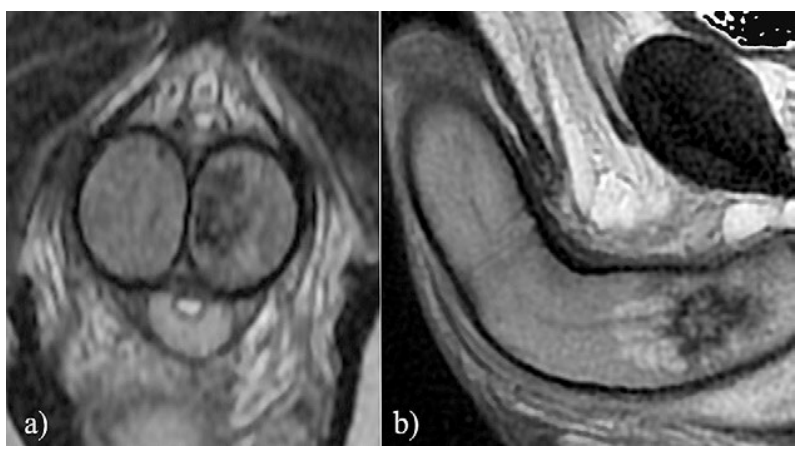

Fig 1. Contrast enhanced magnetic resonance imaging (CEMRI), (a) coronal and (b) sagittal view highlightimg the dilacerations of lacunar tissue at the base of left corpus cavernosum.

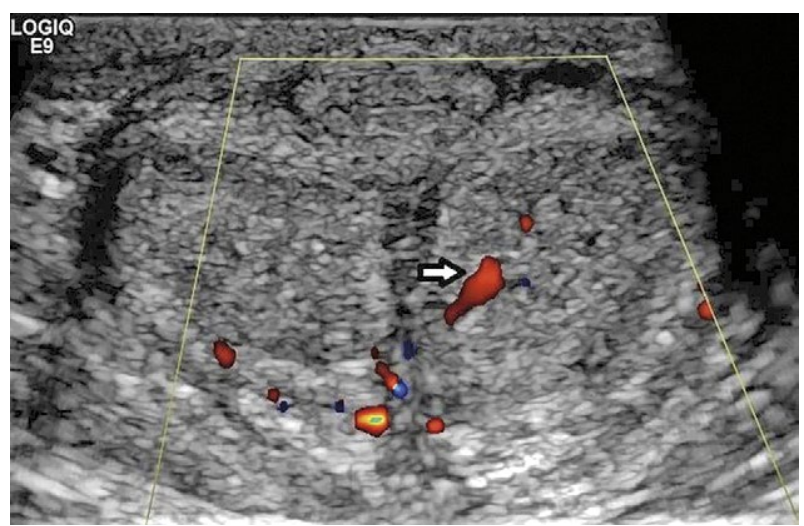

Fig 2. Transverse section reveals at the level of the left cavernous body an arterial signal evidenced by the Doppler CFM (arrow).

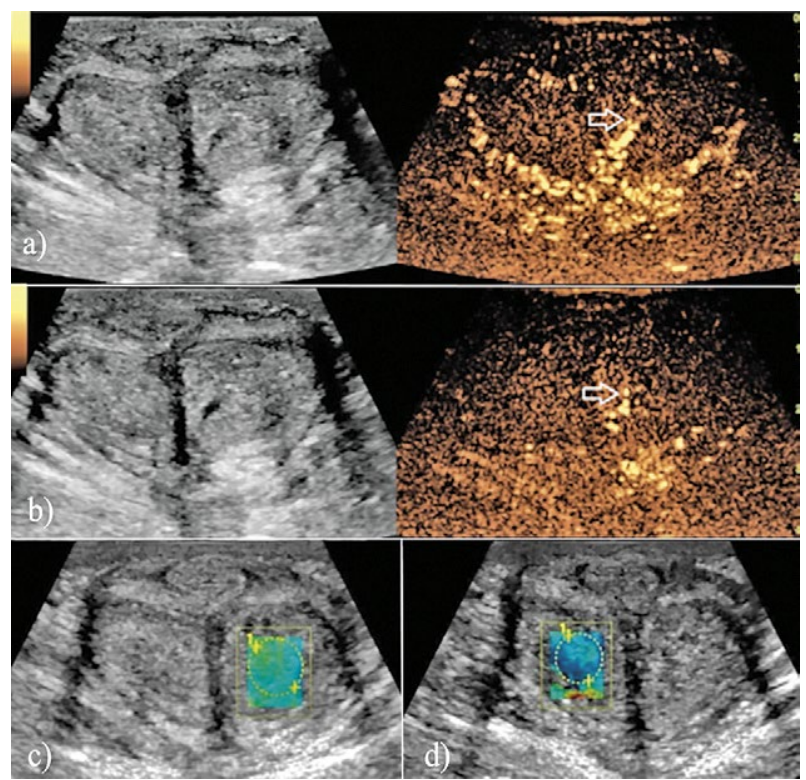

Fig 3. a) CEUS: in the arterial phase, enhancement of the contrast is highlighted at the corresponding level of the arterial signal showed by CFM. In dynamic mode, an apparent turbulent flow is present that suggests a possible "blind" vascular tract inside the left cavernous body (arrow); b) at 120 seconds the CEUS aspect inside the left cavernous body remains persistent but at a low intensity (arrow). Comparative SWE evaluation between c) the left and d) right cavernous body highlights a fibrous reshaping process by non-homogeneous pattern: red spot on a green background versus a contralateral homogenous blue pattern.

Clinical follow-up at 4 weeks, revealed a progressive and significant reduction of the penile erection. Urinary function was normal, but erectile dysfunction was already present. Imaging by US with CFM evaluation highlighted the fibrous tissue at the dilaceration area without any adjacent complications. 


\section{Discussion}

Priapism has a low incidence in the general population with only $0.5-0.9$ cases $/ 100,000$ person-years [4,5], but there is a high prevalence in patients with sickle cell disease (up to $42 \%$ in patients older than 18 years) [6]. From 2015, the European Urology Association (EAU) released the first guide regarding the treatment and diagnosis of priapism [3]. Selective arterial embolization is the main recommendation for treatment in arterial priapism [3], but the lack of experience in this procedure for an interventional radiologist makes it difficult to implement into practice. Surgical approach with ligation of tributary pudendal artery has a low chance as a curative solution. Due to the fact that $78 \%$ of patients with prolonged priapism (more than 7 days) will have erectile dysfunction, early intervention is mandatory for maximizing the therapeutic results [7].

Diagnosis is essential for efficient therapy in this disease. History of the pain and clinical aspect remains the main "instrument" for diagnosis. However, details in order to proceed to minimal invasive techniques relies on imaging. MRI has a good accuracy, but experience of radiologists and limited accessibility is a challenge. US is very accessible and in connection with clinical evaluation is, in many situations, sufficient for the final diagnosis. The procedure is accurate for the detection of morphologic and vascular alteration. Doppler US provides qualitative and quantitative information regarding the vascular blood flow, but CEUS highlights the vascular perfusion pattern at a superior, more accurate level of diagnosis.
In conclusion, CEUS represents alongside gray-scale and Doppler CFM evaluation, an accurate, reproducible, non-invasive and affordable method for assessing tissue and vascular lesions of the cavernous bodies (in an emergency situation and at subsequent follow-up). From our knowledge, this is the first published cases of priapism investigated by CEUS.

\section{References}

1. Montague DK, Jarow J, Broderick GA, et al. American Urological Association guideline on the management of priapism. J Urol 2003;170:1318-1324.

2. Broderick GA, Kadioglu A, Bivalacqua TJ, Ghanem H, Nehra A, Shamloul R. Priapism: pathogenesis, epidemiology, and management. J Sex Med 2010;7:476500 .

3. Salonia A, Eardley I, Giuliana F, et al. European Association of Urology Guidelines on Priapism. Eur Urol 2014;65:480-489.

4. Eland IA, van der Lei J, Stricker BH, Sturkenboom MJ. Incidence of priapism in the general population. Urology 2001;57:970-972.

5. Kulmala RV .Lehtonen TA, Tammela TL. Priapism, its incidence and seasonal distribution in Finland. Scand J Urol Nephrol 1995;29:93-96.

6. Emond AM, Holman R, Hayes RJ, Serjeant GR. Priapism and impotence in homozygous sickle cell disease. Arch Intern Med 1980;140:1434-1437.

7. Kulmala RV, Lehtonen TA, Tammela TL. Preservation of potency after treatment for priapism. Scand J Urol Nephrol 1996;30:313-316. 